T. Ogawa and T. Suzuki

Nagoya Math. J.

Vol. 138 (1995), 33-50

\title{
MICROSCOPIC ASYMPTOTICS FOR SOLUTIONS OF SOME SEMILINEAR ELLIPTIC EQUATIONS
}

\author{
TAKAYOSHI OGAWA AND TAKASHI SUZUKI
}

\section{Dedicated to the memory of Professor Jongsik Kim}

\section{Introduction}

In our previous work [8], we picked up the elliptic equation

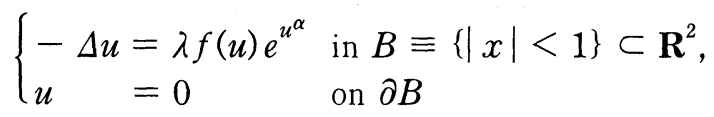

with the nonlinearity $f(u) \geq 0$ in $C^{1}$. We studied the asymptotics of the family $\{(\lambda, u(x))\}$ of classical solutions satisfying

$$
\lambda \downarrow 0 \text { and }\|u\|_{L^{\infty}} \rightarrow+\infty .
$$

Taking the result by Gidas-Ni-Nirenberg [5] into account, we may assume that the solution is radially symmetric and decreasing in $r=|x|$, i.e.,

$$
u=u(|x|) \geq 0, u_{\tau}<0(0<r=|x| \leq 1) .
$$

Furthermore, the coefficient nonlinear term $f(u)$ is supposed to have the polynomial growth. More precisely,

$$
\begin{aligned}
& f^{\prime}(u) \geq 0(u \gg 1), \\
& \lim _{u \rightarrow+\infty}(\log f)^{\prime}(u)=0,
\end{aligned}
$$

and for some $k \in \mathbf{R}$,

$$
0<\liminf _{u \rightarrow+\infty} f(u) u^{-k+\alpha-1} \leq \limsup _{u \rightarrow+\infty} f(u) u^{-k+\alpha-1}<+\infty .
$$

First, the global asymptotics is stated as follows.

Received December 13, 1993. 
Proposition 1 ([8]). Let $(u, \lambda)$ be a family of solutions of (1) with (2).

1. If $0<\alpha<1$, then for any $x \in B, u(x) \rightarrow+\infty$ as $\lambda \rightarrow 0$.

2. If $\alpha>1$, then $u(x) \rightarrow 0$ for any $x \in B \backslash\{0\}$ as $\lambda \rightarrow 0$.

It is well-known that the solutions are expressed explicitly if $f(u) \equiv 1$ and $\alpha$ $=1$. In this case the singular limit is explicitly determined as

$$
u(x) \rightarrow 4 \log \frac{1}{|x|} \text { as } \lambda \downarrow 0 .
$$

Thus the exponent $\alpha=1$ is the borderline of the global asymptotics.

Incidentally, the number of solutions for $f(u)=1$ and $\alpha=1$ is 0,1 , and 2 according to $\lambda>2, \lambda=2$, and $0<\lambda<2$, respectively. The unique solution for

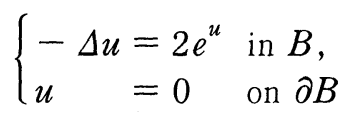

is given as

$$
u(x)=2 \log \frac{2}{1+|x|^{2}} .
$$

This function plays an important role in microscopic asymptotics in the following. Henceforth, we suppose that $\alpha>1$.

Proposition 2 ([8]). Passing to a subsequence, it holds that

$$
u^{\alpha}\left(e^{-\tau / 2} y\right)=u^{\alpha}\left(e^{-\tau / 2}\right)+2 \log \frac{2}{1+|y|^{2}}+o(1)
$$

locally uniformly in $y \in \mathbf{R}^{2} \backslash\{0\}$, where $\tau \rightarrow+\infty$ is taken appropriately.

The purpose of the present paper is to study the uniformity of (4). When the exponent is in $1<\alpha<2$, the following fact has proven in [8] with the aid of o.d.e. approach by Atkinson-Peletier [2].

Proposition 3 ([8]). In the case of $f(u) \equiv 1$ and $1<\alpha<2$, the uniform convergence in (4);

$$
\sup _{|y| \leq e^{\tau / 2}}\left|u^{\alpha}\left(e^{-\tau / 2} y\right)-u^{\alpha}\left(e^{-\tau / 2}\right)-2 \log \frac{2}{1+|y|^{2}}\right| \rightarrow 0
$$

never holds for any $\{\tau\}$. 
In case of $\alpha>2$, it is not known whether classical solutions for (1) with (2) exist or not ([1], [2]). The exponent $\alpha=2$ is considered to be a borderline for the existence. What we want to claim here is that it is also the borderline from the microscopic asymptotic point of view. We shall give a uniform convergence result for this borderline case. The following theorem is the main result of the present paper.

THEOREM 4. If $\alpha=2$ and

$$
E_{0} \equiv \limsup _{\lambda \rightarrow 0} \int_{B}|\nabla u|^{2} d x<6 \pi
$$

then the convergence (4) is locally uniform in $y \in \mathbf{R}^{2}$. In other words, the uniform asymptotics near $y=0$ is exactly expressed as in (4).

Concerning the existence of such a family, we have the following theorem.

Theorem 5. If $k>2$ in (3), there exists a family $\{(\lambda, u(x))\}$ of classical solutions of (1) with $\alpha=2$, satisfying (2) and

$$
E \equiv \int_{B}|\nabla u|^{2} d x \rightarrow 4 \pi
$$

In case of $\alpha<2, E_{0}<\infty$ implies that

$$
\lambda f(u) e^{u^{\alpha}} \in L^{1+\varepsilon}(\Omega)
$$

for some $\varepsilon>0$ because of the Trudinger-Moser inequality ([11], [7]) i.e.

$$
\sup _{v}\left\{\int_{\Omega} e^{v^{2}} d x \mid\|\nabla v\|_{2}^{2} \leq 4 \pi\right\} \leq C|\Omega| .
$$

Consequently the blow-up (2) does not occur by the standard elliptic estimates. In this sense, Theorems 4 and 5 are peculiar to the case $\alpha=2$.

A similar observation also yields for the case $\alpha=2$, that

$$
\liminf _{\lambda \rightarrow 0} \int_{B}|\nabla u|^{2} d x \geq 4 \pi
$$

for the solution of (1) with (2). We shall give a more specified estimate (Lemma 8) for the Dirichlet integral for the solution by using the scaling parameter which will be a key estimate to show Theorem 4 .

The special case 


$$
-\Delta u=\lambda u e^{u^{2}}, u>0 \text { in } B=\{|x|<1\} \subset \mathbf{R}^{2}
$$

with

$$
u=0 \text { on } \partial B
$$

is closely related to the Trudinger-Moser inequality and also Carleson-Chang's theorem ([4], see also [6], [10]). However, this case of $k=2$ is not treated in Theorem 5. We shall pick up such a kind of equations in a forthcoming paper.

For the proof of Theorem 4, we invoke the following uniform estimate by Brezis-Merle [3]. Assume that $\Omega \subset \mathbf{R}^{2}$ is a bounded domain. Consider a family of solutions to

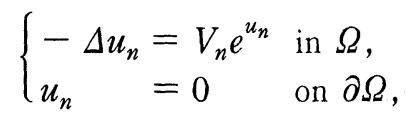

where $\left\{V_{n}\right\}$ is a given family of functions on $\Omega$.

Lemma 6 ([3]). Let $\left\{V_{n}\right\}$ be given functions with

$$
\left\|V_{n}\right\|_{L^{p}(\Omega)} \leq \beta
$$

for some $1<p \leq \infty$ and $u_{n}$ be a solution of (8) in the sense of distribution. Suppose that

$$
\int_{\Omega}\left|V_{n}\right| e^{v_{n}} d x \leq \gamma<\frac{4 \pi}{p^{\prime}}, p^{\prime}=p / p-1
$$

then the solution $u_{n}$ is bounded independent of $n$, i.e.,

$$
\left\|v_{n}\right\|_{L^{\infty}} \leq C(\beta, \gamma, \Omega, p)
$$

The smallness assumption (5) in Theorem 4 comes from the assumption (9).

The proof of Theorem 4 is based on that of Proposition 2. In $\S 2$ we shall review the latter to perform the former in $\S 3$. The proof of Theorem 5 is independent and shall be given in $\S 4$.

\section{Summary of the Proof of Proposition 2}

We take the case $\alpha=2$ for simplicity. Namely, we consider the smooth solution $u$ of

$$
\left\{\begin{aligned}
-\Delta u & =\lambda f(u) e^{u^{2}} & & \text { in } B \\
u & =0 & & \text { on } \partial B
\end{aligned}\right.
$$


where $f(u) \geq 0$ is a $C^{1}$ function satisfying (3).

The solution $u$ becomes radially symmetric and has the property that

$$
u_{r}<0(0<r=|x| \leq 1) \text {. }
$$

We put the scaling solution $v(r)$ as

$$
v(r)=u^{2}(\gamma r)-u^{2}(\gamma)
$$

for some scaling constant $\gamma \rightarrow 0$. This function $v$ is subject to

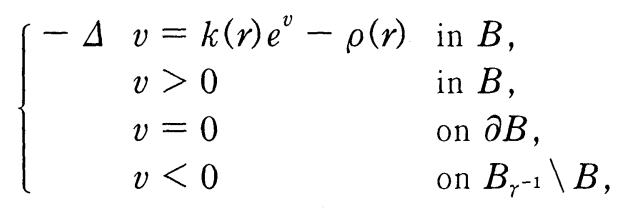

where we set

$$
\left\{\begin{array}{l}
k(r)=2 \lambda u(\gamma r) f(u(\gamma r)) e^{u(\gamma)} \gamma^{2}, \\
\rho(r)=2 \gamma^{2}|\nabla u(\gamma r)|^{2}
\end{array}\right.
$$

and $B_{r^{-1}}=\left\{x \in \mathbf{R}^{2},|x|<\gamma^{-1}\right\}$.

Writing both equations (10) and (12) into the ODE form, we introduce the transformation $r=e^{-t / 2}, U(t)=u(r)$ and $V(t)=v(r)$ to get

$$
\left\{\begin{aligned}
\ddot{U}+ & \frac{\lambda}{4} f(U) e^{U^{2}-t}=0, \\
U & >0(t>0), \\
& \dot{U}>0(t>0), \\
& \dot{U} e^{t / 2} \rightarrow 0(t \rightarrow+\infty)
\end{aligned}\right.
$$

and

$$
\left\{\begin{aligned}
\ddot{V}+ & \frac{1}{4} K(t) e^{V(t)-t}-2 \dot{U}_{\tau}^{2}(t)=0, \\
& V>0(t>0), \\
& \dot{V}>0(t>0) \\
& \dot{V} e^{t / 2} \rightarrow 0(t \rightarrow+\infty),
\end{aligned}\right.
$$

where $\tau=-2 \log \gamma, U_{\tau}(t)=U(t+\tau)$ and $K(t) \equiv 2 \lambda U_{\tau} f\left(U_{\tau}\right) e^{U(\tau)^{2}-\tau}=k(r)$.

The equation (15) has a representation of the integral equation as

$$
V(t)-V(+\infty)+\int_{t}^{\infty}(s-t) \frac{K(s)}{4} e^{V(s)-s} d s=2 \int_{t}^{\infty}(s-t) U_{\tau}^{2}(s) d s .
$$


In the proof of Proposition 1 (cf. [8]), the asymptotics

$$
\eta \equiv \max _{0 \leq r \leq 1}\left|r u_{r}\right| \rightarrow 0
$$

is proven. Since

$$
\dot{U}_{\tau}(t)=-\left.\frac{1}{2} r u_{r}\right|_{r=\exp \left(-\frac{t+r}{2}\right)},
$$

it holds that

$$
\left\|\dot{U}_{\tau}\right\|_{L^{\infty}(-\tau, \infty)} \rightarrow 0
$$

This relation deduces that

$$
K(t) \rightarrow \text { constant, locally uniformly in } t \in \mathbf{R} .
$$

Two cases should be distinguished for the parameter $\tau \rightarrow+\infty$ to be specified. Let

$$
\begin{aligned}
m & =\max _{0 \leq r \leq 1} 2 \lambda u(r) f(u(r)) e^{u^{2}(r)} r^{2} \\
& =\sup _{t \in \mathbf{R}} 2 \lambda U(t) f(U(t)) e^{U^{2}(t)-t} .
\end{aligned}
$$

Case 1: $m \rightarrow+\infty$

In this case we can take $\tau \rightarrow+\infty$ as

$$
K(0)=2 .
$$

The asymptotics (18) and (19) imply that

$$
\rho \rightarrow 0, k \rightarrow 2 \text { locally uniformly in } \mathbf{R}^{2} \backslash\{0\} .
$$

The relations (12) is reduced to

$$
\|-\Delta v\|_{L^{\infty}(1<|y|<R)}=O(1)
$$

with

$$
v=0 \text { on } \mid y=1
$$

for any $R>1$. Hence $\{v\}$ never blows-up on $\{|y| \geq 1\}$.

On the other hand, by (22), the boundedness of the equation (12) near $\partial B$ follows and this implies

$$
\left\|v_{r}\right\|_{L^{\infty}(\partial B)} \leq C
$$


Therefore, for any $\varepsilon>0$,

$$
\begin{aligned}
\int_{B / B_{\varepsilon / 2}}\left\{k(r) e^{v}\right. & -\rho(r)\} d x \\
& =\int_{B / B_{\varepsilon / 2}}(-\Delta v) d x \\
& \leq-\omega_{2} v_{r}(1) \leq C .
\end{aligned}
$$

While by (22),

$$
\begin{aligned}
\int_{B / B_{\varepsilon / 2}} & \left\{k(r) e^{v}-\rho(r)\right\} d x \\
\geq & \int_{B / B_{\varepsilon / 2}}\left\{\frac{1}{2} e^{v}-\frac{1}{4}\right\} d x \\
\geq & \pi \int_{\varepsilon / 2}^{\varepsilon} v(r) r d r \\
\leq & C \varepsilon^{2} v(\varepsilon) .
\end{aligned}
$$

Hence we obtain an apriori estimate for $v$ on $\mathbf{R}^{2} \backslash\{0\}$. Together with the equation (12), we may obtain the limit function $v_{0}$ as

$$
v(r) \rightarrow v_{0}(r) \text { locally uniformly on } \mathbf{R}^{2} \backslash\{0\}
$$

by the Ascori-Arzela theorem.

Finally, the singular limit $v_{0}(y)=2 \log \frac{2}{1+|y|^{2}}$ is specified through

$$
-\Delta v_{0}=2 e^{v_{0}} \text { in } \mathbf{R}^{2} \backslash\{0\}
$$

and

$$
v_{0} \geq 0 \text { on }|y| \leq 1
$$

Case 2: $m=O(1)$

In this case, we choose $r \rightarrow+\infty$ by

$$
U^{2}(+\infty)=U^{2}(\tau)+2 \log 2 .
$$

The condition $m=O(1)$ implies that

$$
\|K\|_{L^{\infty}(-\tau, \infty)}=O(1)
$$

and hence by passing to a subsequence,

$$
K(0) \rightarrow 2 \mu
$$


for some $\mu \geq 0$.

From (19), it follows the convergence

$$
K(t) \rightarrow 2 \mu \text { locally uniformly in } t \in(-\infty,+\infty),
$$

while

$$
\|v\|_{L^{\infty}\left(|y|<e^{\tau / 2)}\right.}=2 \log 2
$$

holds by (23). Utilizing the elliptic estimate, we see that a subsequence of $\{v\}$ converges locally uniformly in $\mathbf{R}^{2} \backslash\{0\}$. The limiting function $v_{0}(y)$ satisfies

$$
\begin{gathered}
-\Delta v_{0}=2 \mu e^{v_{0}} \text { in } \mathbf{R}^{2}, \\
v_{0}=0 \text { on }|y|=1
\end{gathered}
$$

and

$$
\left\|v_{0}\right\|_{L^{\infty}}=v_{0}(0) \leq 2 \log 2 \text {. }
$$

The conclusion $v_{0}(y)=2 \log \frac{2}{1+|y|^{2}}$ follows from $\mu=1$ or equivalently $v_{0}(0)=2 \log 2$. However, the right-hand side of (16) is non-negative and $V(+\infty)=2 \log 2$. Therefore, the dominated convergence theorem implies that

$$
\begin{gathered}
0 \leq V_{0}(t)+\int_{t}^{\infty}(s-t) \frac{\mu}{2} e^{V_{0}(s)-s} d s-2 \log 2 \\
=V_{0}(+\infty)-2 \log 2
\end{gathered}
$$

for $V_{0}(t)=v_{0}(r)$, or equivalently,

$$
v_{0}(0) \geq 2 \log 2 .
$$

This completes the proof.

We note that the relation

$$
\lambda u\left(e^{-\tau / 2}\right) f\left(u\left(e^{-\tau / 2}\right)\right) e^{u^{2}\left(e^{-\tau / 2}\right)-\tau}=1+o(1)
$$

follows from the proof.

\section{Proof of Theorem 4}

We have to prepare a few lemmas. 
Lemma 7. The function $k(|y|)$ defined by (13) satisfies that

$$
\|k\|_{L^{p}(|y|<1)}=O(1) \text { for } 1<p<\infty .
$$

Proof. In the case of $m=O(1)$, the uniform estimate (24) holds. Therefore, we have only to consider the case $m \rightarrow+\infty$.

Then, $\tau \rightarrow+\infty$ is determined through (21), i.e.,

$$
2=K(0)=2 \lambda U(\tau) f(U(\tau)) e^{U^{2}(\tau)-\tau} .
$$

Hence

$$
\begin{aligned}
K(t) & =2 \lambda U_{\tau}(t) f\left(U_{\tau}(t)\right) e^{U^{2}(\tau)-\tau} \\
& =2 \frac{U_{\tau}(t) f\left(U_{\tau}(t)\right)}{U(\tau) f(U(\tau))} \approx\left(\frac{U_{\tau}(t)}{U(\tau)}\right)^{k}
\end{aligned}
$$

by (3).

Writing

$$
\frac{U_{\tau}(t)}{U(\tau)}=1+\frac{1}{U(\tau)} \int_{0}^{t} \dot{U}_{\tau}(s) d s
$$

we reach

$$
0 \leq \frac{U_{\tau}(t)}{U(\tau)} \leq C(1+t) \quad(t \geq 0)
$$

by (18). The conclusion (25) follows from (26), (27), and

$$
k(r)=K(t) \text { for } r=e^{-t / 2} .
$$

Lemma 8. For any fixed $R>0$, we have

$$
4 \pi \leq \liminf _{\lambda \rightarrow 0} \int_{\left\{R e^{-\tau / 2}<|x|<1\right\}}|\nabla u|^{2} d x .
$$

Proof. As is described in the previous section,

$$
V(t) \rightarrow V_{0}(t) \text { locally uniformly in } t \in(-\infty,+\infty)
$$

for

$$
V_{0}(t)=2 \log \frac{2}{1+e^{-t}}
$$


Making use of the elliptic estimate in (15), this implies that

$$
\dot{V}(t) \rightarrow \dot{V}_{0}(t) \text { locally uniformly in } t \in(-\infty,+\infty) \text {. }
$$

Here,

$$
\dot{V}(t)=2 U(t+\tau) \dot{U}(t+\tau)
$$

and

$$
\dot{V}_{0}(t)=\frac{2 e^{-t}}{1+e^{-t}} .
$$

Therefore, writing $R=e^{-t / 2}$, we obtain

$$
-R e^{-\tau / 2} u\left(R e^{-\tau / 2}\right) u_{r}\left(R e^{-\tau / 2}\right) \rightarrow \frac{2 R^{2}}{1+R^{2}} .
$$

The equation (10) deduces that

$$
\begin{gathered}
\int_{\left\{R e^{-\tau / 2}<|x|<1\right\}}|\nabla u|^{2} d x=-2 \pi R e^{-\tau / 2} u\left(R e^{-\tau / 2}\right) u_{r}\left(R e^{-\tau / 2}\right) \\
+\int_{R e^{-\tau / 2}<|x|<1} \lambda u f(u) e^{2} d x .
\end{gathered}
$$

Therefore, combining (29) and (30), we see for $\tilde{R}>R$

$$
\begin{aligned}
\frac{4 \pi \tilde{R}^{2}}{1+\tilde{R}^{2}} & \leq \liminf _{\lambda \rightarrow 0} \int_{\left\{\tilde{R} e^{-\tau / 2}<|x|<1\right\}}\left(|\nabla u|^{2}-\lambda u f(u) e^{u^{2}}\right) d x \\
& \leq \liminf _{\lambda \rightarrow 0} \int_{\left\{\tilde{R} e^{-\tau / 2}<|x|<1\right\}}|\nabla u|^{2} d x \\
& \leq \liminf _{\lambda \rightarrow 0} \int_{\left\{R e^{-\tau / 2}<|x|<1\right\}}|\nabla u|^{2} d x .
\end{aligned}
$$

By taking $\tilde{R}$ arbitrarily large, we obtain (28).

\section{LEMMA 9. Under the assumption of}

$$
\limsup _{\lambda \rightarrow 0} \int_{B}|\nabla u|^{2} d x<6 \pi,
$$

we have

$$
\|v\|_{L^{\infty}(|y|<R)}=O(1)
$$

for any $R>0$. Here, the function $v(|y|)$ is defined by (11). 
Proof. We prove this lemma by the aid of Lemma 6 in Section 1.

The estimates (28) and (32) imply that

$$
\limsup _{\lambda \rightarrow 0} \int_{\left\{|x|<R e^{-\tau / 2\}}\right.}|\nabla u|^{2} d x<2 \pi \text { for any } R>0 .
$$

Hence the function $\rho(|y|)$ introduced in (13) satisfies that

$$
\limsup _{\lambda \rightarrow 0}\|\rho\|_{L^{1}(|y|<1)}=4 \pi \int_{0}^{\gamma^{-1}}|\nabla u(r)|^{2} d r<4 \pi .
$$

We may suppose that $0<R \ll 1$ in showing (33). Let us take the functions $h_{1}$ and $h_{2}$ as

$$
-\Delta h_{1}=0 \quad \text { in }|y|<R, \quad h_{1}=v \quad \text { on }|y|=R
$$

and

$$
-\Delta h_{2}=-\rho \quad \text { in }|y|<R, \quad h_{2}=0 \quad \text { on }|y|=R .
$$

We have already proven that

$$
\|v\|_{L^{\infty}(|y|=R)}=O(1)
$$

so that

$$
\left\|h_{1}\right\|_{L^{\infty}(|y|<1)}=O(1)
$$

holds by the maximum principle. On the other hand, $\rho \geq 0$ and hence

$$
h_{2} \leq 0 \text { in }|y|<R \text {. }
$$

This implies the estimate

$$
\left\|e^{h}\right\|_{L^{\infty}(|y|<R)}=O(1)
$$

for

$$
h=h_{1}+h_{2} .
$$

Because of (12), the function $w=v-h$ solves that

$$
\begin{aligned}
-\Delta w & =-\Delta v+\Delta h=-\Delta v+\rho \\
& =k e^{v}=k e^{h} e^{w} \text { in }|y|<R
\end{aligned}
$$

and

$$
w=0 \text { on }|y|=R
$$


Here, Lemma 7 and (37) are utilized to deduce

$$
\left\|k e^{h}\right\|_{L^{p}(|y|<R)}=O(1) \text { for } 1<p<\infty .
$$

On the other hand we have

$$
\begin{aligned}
\left\|k e^{h} e^{w}\right\|_{L^{1}(|y|<R)} & =\left\|k e^{v}\right\|_{L^{1}(|y|<R)} \\
& =\|\rho\|_{L^{1}(|y|<R)}+\int_{\{|y|<R\}}(-\Delta v) d x
\end{aligned}
$$

by (12) and $k, \rho \geq 0$. By Proposition 2, we have

$$
v_{r}(R)=v_{0 r}(R)+o(1) .
$$

Therefore, from (40) and (29) we obtain

$$
\begin{aligned}
\left\|k e^{h} e^{w}\right\|_{L^{1}(|y|<R)} & =\|\rho\|_{L^{1}(|y|<R)}-2 \pi R v_{r}(R) \\
& \leq\|\rho\|_{L^{1}(|y|<1)}-2 \pi R v_{0 r}(R)+o(1) \\
& \leq\|\rho\|_{L^{1}(|y|<1)}+\frac{4 \pi R^{2}}{1+R^{2}}+o(1)
\end{aligned}
$$

for $0<R<1$.

Here, we take $R>0$ sufficiently small to deduce that

$$
\limsup _{\lambda \rightarrow 0}\left\|k e^{h} e^{w}\right\|_{L^{1}(|y|<R)}<4 \pi
$$

by (34).

Now, we can apply Lemma 6 for (38) with (29). Then it follows that

$$
\|w\|_{L^{\infty}(|y|<R)}=O(1) .
$$

However, we have from (36),

$$
0 \leq v=w+h_{1}+h_{2} \leq w-h_{1} \text { in }|y|<R .
$$

Consequently (33) follows from (35) and (41).

We are in position to complete the proof of Theorem 4.

Proof of Theorem 4. As we have shown,

$$
v \rightarrow v_{0} \text { locally uniformly in } \mathbf{R}^{2} \backslash\{0\}
$$

so that 


$$
V_{0}(+\infty)=2 \log 2 \leq \liminf _{\lambda \rightarrow 0} V(+\infty)
$$

Furthermore,

$$
K(t) \rightarrow 2, V(t) \rightarrow V_{0}(t) \text { locally uniformly in }(-\infty,+\infty)
$$

and also

$$
\|V\|_{L^{\infty}\left(t_{1}, \infty\right)}=O(1) \text { for any } t_{1} \in \mathbf{R}
$$

by Lemma 9 . Finally, we have

$$
|K(t)| \leq C(1+t)^{m} \text { for } t \gg 1
$$

from (26) and (27).

Here, the dominated convergence theorem is utilized to take the limit in (16). We obtain

$$
\begin{aligned}
0 & \leq \liminf _{\lambda \rightarrow 0} \int_{t}^{\infty} 2(s-t) \dot{U}_{\tau}^{2}(s) d s \leq \limsup _{\lambda \rightarrow 0} \int_{t}^{\infty} 2(s-t) \dot{U}_{\tau}^{2}(s) d s \\
& \leq V_{0}(t)+\int_{t}^{\infty} \frac{1}{2}(s-t) e^{V_{0}(s)-s} d s-\liminf _{\lambda \rightarrow 0} V(+\infty) \\
& =V_{0}(+\infty)-\liminf _{\lambda \rightarrow 0} V(+\infty) \leq 0
\end{aligned}
$$

by (42). Therefore,

$$
\int_{t}^{\infty} 2(s-t) \dot{U}_{\tau}^{2}(s) d s \rightarrow 0 \quad(t \in \mathscr{R})
$$

Furthermore,

$$
\begin{aligned}
0 & =\lim _{\lambda \rightarrow 0} \int_{t}^{\infty} 2(s-t) \dot{U}_{\tau}^{2}(s) d s \\
& =V_{0}(t)+\int_{t}^{\infty} \frac{1}{2}(s-t) e^{V_{0}(s)-s} d s-\lim _{\lambda \rightarrow 0} V(+\infty) \\
& =V_{0}(+\infty)-\lim _{\lambda \rightarrow 0} V(+\infty) .
\end{aligned}
$$

Hence

$$
V(+\infty) \rightarrow V_{0}(+\infty)
$$

Going back to (16), we have

$$
\left|V(t)-V_{0}(t)\right| \leq \int_{t}^{\infty}(s-t) 2 \dot{U}_{\tau}^{2}(s) d s+\left|V(+\infty)-V_{0}(+\infty)\right|
$$




$$
+\int_{t}^{\infty}(s-t)\left|\frac{K(s)}{4} e^{V(s)}-\frac{1}{2} e^{V_{0}(s)}\right| e^{-s} d s
$$

so that

$$
\begin{aligned}
\sup _{t \geq t_{1}} V(t)-V_{0}(t) \mid \leq \int_{t_{1}}^{\infty} & \left(s-t_{1}\right) 2 \dot{U}_{\tau}^{2}(s) d s+\left|V(+\infty)-V_{0}(+\infty)\right| \\
& +\int_{t_{1}}^{\infty}\left(s-t_{1}\right)\left|\frac{K(s)}{4} e^{V(s)}-\frac{1}{2} e^{V_{0}(s)}\right| e^{-s} d s,
\end{aligned}
$$

where $t_{1} \in \mathscr{R}$.

The first two terms converges to zero by (46) and (47). For the last term, we utilize (43)-(45) and the dominated convergence theorem. Thus we obtain

$$
\left\|V-V_{0}\right\|_{L^{\infty}\left(t_{1}, \infty\right)} \rightarrow 0,
$$

which means that

$$
v \rightarrow v_{0} \text { locally uniformly in } \mathbf{R}^{2},
$$

the desired convergence.

\section{Proof of Theorem 5}

The Trudinger-Moser inequality mentioned in Section 1 is expressed as

$$
\sup _{v}\left\{\int_{B} e^{v^{2}} d x \mid\|\nabla v\|_{2}^{2} \leq 4 \pi\right\} \leq C|\Omega|
$$

The constant $4 \pi$ in (48) is shown to be best possibly by [7]. The following proposition is a slight refinement.

Proposition 10. For any continuous function $k(u) \geq 0$ with

$$
\lim _{u \rightarrow+\infty} k(u)=+\infty,
$$

there exists a family $\{w\} \subset H_{0}^{1}(B)$ satisfying

$$
w \geq 0, \int_{B}|\nabla w|^{2} d x<4 \pi
$$

and

$$
\int_{B} k(w) e^{w^{2}} d x \rightarrow+\infty
$$


This fact is combined with the following lemma proven by Shaw via the Lagrange multiplier principle.

LEMma 11 ([9]). Suppose the existence of a non-negative function $w \in$ $H_{0}^{1}(B)$ such that

$$
\int_{B}|\nabla w|^{2} d x=\gamma<4 \pi
$$

Then, there exists a solution $(\lambda, u(x))$ for (10) such that

$$
\int_{B} G(u) d x=\int_{B} G(w) d x
$$

and

$$
\int_{B}|\nabla u|^{2} d x \leq \gamma
$$

where

$$
G(u)=\int_{0}^{u} f(u) e^{u^{2}} d u .
$$

The condition (3) with $k>2$ implies that

$$
\lim _{u \rightarrow+\infty} f(u) / u=+\infty
$$

If

$$
G(u)=k(u) e^{u^{2}}
$$

this means that

$$
\lim _{u \rightarrow+\infty} k(u)=+\infty
$$

Hence Proposition 10 and Lemma 11 are applicable.

We get a family $\{(\lambda, u(x))\}$ of solutions for (10) satisfying

$$
\limsup _{\lambda \rightarrow 0} \int_{B}|\nabla u|^{2} d x \leq 4 \pi
$$

and

$$
\int_{B} G(u) d x \rightarrow+\infty
$$


The asymptotics (50) holds only when

$$
\|u\|_{L^{\infty}} \rightarrow+\infty \text {. }
$$

Furthermore, $f^{\prime}(u)>0$ for $u \gg 1$ so that there exists a constant $C>0$ such that

$$
G(u)=\int_{0}^{u} f(u) e^{u^{2}} d u \leq C u f(u) e^{u^{2}}(u \geq 0) .
$$

Therefore,

$$
\begin{gathered}
\lambda \int_{B} G(u) d x \leq C \int_{B} \lambda u f(u) e^{u^{2}} d x \\
=C \int_{B}|\nabla u|^{2} d x=O(1)
\end{gathered}
$$

by (49) and hence

$$
\lambda \downarrow 0
$$

by $(50)$.

In this way Theorem 5 has been reduced to Proposition 10. For the sake of completeness we show the proof, although it is quite similar to [7].

Proof of Proposition 10. The family is constructed from $W(t)=w(r)$ for $r=$ $e^{-t / 2}$. We have

$$
\int_{0}^{\infty} \dot{W}^{2} d t=\frac{1}{4 \pi} \int_{\beta}|\nabla w|^{2} d x
$$

and

$$
\int_{0}^{\infty} k(W) e^{W^{2}-t} d t=\frac{1}{\pi} \int_{\beta} k(w) e^{w^{2}} d x
$$

Therefore, the desired relations are reduced to

$$
\begin{gathered}
(W) \subset A C[0, \infty), W(0)=0, W \geq 0, \\
\int_{0}^{\infty} \dot{W}^{2} d t<1
\end{gathered}
$$

and

$$
\int_{0}^{\infty} k(W) e^{W^{2}-t} d t \rightarrow+\infty
$$


where $A C$ denotes the set of absolutely continuous functions.

Taking $\varepsilon$ sufficiently small, we put

$$
\eta_{\varepsilon}(s)=\min (s, 1-\varepsilon)
$$

and

$$
W(t)=\varepsilon^{-1 / 2} \eta_{\varepsilon}(\varepsilon t) .
$$

For this function, the requirement (51) is obvious. The inequality (52) is examined as

$$
\int_{0}^{\infty} \dot{W}^{2} d t=\int_{0}^{\infty} \eta_{\varepsilon}^{\prime}(s)^{2} d s=(1-\varepsilon)^{2}<1
$$

Finally, we conclude that

$$
\begin{gathered}
\int_{0}^{\infty} k(W) e^{W^{2}-t} d t=\int_{0}^{\infty} k\left(\varepsilon^{-1 / 2} \eta \varepsilon(s)\right) e^{\varepsilon^{-1} \eta_{\varepsilon}-\eta^{-1} s} \eta^{-1} d s \\
\geq k\left(\eta^{-1 / 2}(1-\varepsilon)\right) \int_{1-\eta}^{\infty} e^{(1-\varepsilon-s) \varepsilon^{-1}} \varepsilon^{-1} d s \\
=k\left(\varepsilon^{-1 / 2}(1-\varepsilon)\right) \rightarrow+\infty \text { as } \varepsilon \downarrow 0 .
\end{gathered}
$$

Thus the proof has been completed.

\section{REFERENCES}

[1] Adimurthi, Existence of positive solutions of the semilinear Dirichlet problem with critical growth for the $n$-Laplacian, Ann. Scou. Norm. Sup. Pisa, 17(1990), $393-413$.

[2] Atkinson, F. V., Peletier, L. A., Ground states of $-\Delta u=f(u)$ and the EmdenFowler equation, Arch. Rat. Mech. Anal., 96 (1986), 147-165.

[3] Brezis, H., Merle, F., Uniform estimates and blow-up behavior for solutions of $-\Delta u=V(x) e^{u}$ in two dimensions, Comm. in Partial Differential Equations, 16 (1991), 1223-1253.

[4] Carleson, L., Chang, S-Y. A., On the existence of an extremal function for an inequality of J. Moser, Bull. Sc. Math., 110 (1986), 113-127.

[ 5 ] Gidas, B., Ni, W-M., Nirenberg, L., Symmetry and related properties via the maximum principle, Comm. Math. Phys., 68 (1979), 209-243.

[6] McLeod, J. B., Peletier, L. A., Observation on Moser's inequality, Arch. Rat. Mech. Anal., 106 (1989), 261-285.

17] Moser, J., A sharp form of an inequality by N. Trudinger, Indiana Univ. Math. J., 11 (1971), 1077-1092.

[8] Ogawa, T., Suzuki, T., Nonlinear elliptic equations with critical growth related to the Trudinger inequality, to appear in Asymptotic Analysis. 
[9] Shaw, M. C., Eigenfunctions of the nonlinear equation $\Delta u+\nu f(x, u)=0$ in $\mathbf{R}^{2}$, Pacific J. Math., 129 (1987), 349-356.

[10] Struwe, M., Critical points of $H_{0}^{1, n}$ into Orlicz spaces, Ann. Inst. H. Poincaré Analy. se non linéaire, 5 (1988), 425-464.

[11] Trudinger, N., On imbedding into Orlicz space and some applications, J. Math. Mech., 17 (1967), 473-484.

Takayoshi Ogawa

Graduate School of Polymathematics

Nagoya University,

Furôchô, Chikusa-ku, Nagoya, 464-01, Japan

Takashi Suzuki

Department of Mathematics

Osaka University

Machikaneyamachô, Toyonaka, Osaka 560, Japan 